\title{
Variation in National Use of Long-Term ADT by Disease Aggressiveness Among Men With Unfavorable-Risk Prostate Cancer
}

\author{
Vinayak Muralidhar, MSc $c^{\mathrm{a}}$; Paul J. Catalano, ScD ${ }^{\mathrm{b}}$; Gally Reznor, MSc; Brandon A. Mahal, MD; \\ Toni K. Choueiri, MD; Christopher J. Sweeney, MBBS ; Neil E. Martin, MD, MPH ${ }^{\mathrm{d}}$; Clair J. Beard, MDd; \\ Yu-Wei Chen, MD, MS ${ }^{\mathrm{d}}$; Michelle D. Nezolosky, BA ${ }^{\mathrm{d}}$; Karen E. Hoffman, MD, MHSc, MPH; \\ Felix Y. Feng, MD; ; Quoc-Dien Trinh, MD ${ }^{\mathrm{h}}$; and Paul L. Nguyen, $\mathrm{MD}^{\mathrm{d}}$
}

\begin{abstract}
Background: The current NCCN Clinical Practice Guidelines in Oncology for Prostate Cancer recommend long-term androgen deprivation therapy (ADT) for all men with high-risk prostate cancer treated with external-beam radiation therapy (EBRT). We determined whether the use of longterm ADT varied by the recently defined subcategories of high-risk disease (favorable, other, and very high) versus unfavorable intermediate-risk disease. Methods: We identified 5,524 patients with unfavorable-risk prostate cancer diagnosed from 2004 to 2007 and managed with EBRT using the SEER-Medicare linked database. Patients were stratified by risk group: unfavorable intermediate-risk, favorable high-risk (previously defined and validated as clinical stage T1c, Gleason score of $4+4=8$, and prostate-specific antigen [PSA] level $<10 \mathrm{ng} / \mathrm{mL}$, or clinical stage T1c, Gleason score of 6, and PSA level >20 ng/mL), very-high-risk (clinical stage T3b-T4 or primary Gleason pattern 5), or other high risk (ie, neither favorable nor very high). We used multivariable competing risks regression to estimate the rates of long-term ( $\geq 2$ years) ADT by group. Results: Men with favorable high-risk prostate cancer were significantly less likely to receive long-term ADT than those with other high-risk disease (15.4\% vs $24.6 \%$, adjusted hazard ratio $[A H R], 0.68 ; 95 \% \mathrm{Cl}, 0.60-0.76 ; P<.001)$, and similarly likely as those with unfavorable intermediate-risk disease (AHR, $1.10 ; 95 \% \mathrm{Cl}$, 0.99-1.23; $P=.087)$. Other high-risk disease was less likely to receive long-term ADT than very high-risk cancer $(24.6 \%$ vs $30.8 \% ; A H R, 0.83 ; 95 \%$, 0.74-0.93; $P=.002$ ). Conclusions: Despite current guidelines, patients with EBRT-managed high-risk prostate cancer received significantly different rates of long-course ADT based on subclassification. Our results suggest that oncologists view these patients as a heterogeneous group with favorable high-risk cancer warranting less aggressive therapy than other high-risk or very high-risk disease.
\end{abstract}

J Natl Compr Canc Netw 2016;14(4):421-428

\section{NCCN: Continuing Education}

Accreditation Statement

This activity has been designed to meet the educational needs of physicians and nurses involved in the management of patients with cancer. There is no fee for this article. No commercial support was received for this article. The National Comprehensive Cancer Network (NCCN) is accredited by the ACCME to provide continuing medical education for physicians.

NCCN designates this journal-based CME activity for a maximum of 1.0 AMA PRA Category 1 Credit $^{\mathrm{TM}}$. Physicians should claim only the credit commensurate with the extent of their participation in the activity.

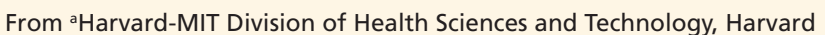
Medical School; 'bepartment of Biostatistics and Computational Biology, Dana-Farber Cancer Institute, Harvard Medical School; and 'Center for Surgery and Public Health, dDepartment of Radiation Oncology, and eDepartment of Medical Oncology, Brigham and Women's Hospital/DanaFarber Cancer Institute, Harvard Medical School, Boston, Massachusetts; fDepartment of Radiation Oncology, MD Anderson Cancer Center, Houston, Texas; ' Department of Radiation Oncology, University of Michigan, Ann Arbor, Michigan; and hepartment of Urology, Brigham and Women's Hospital, Boston, Massachusetts.

Submitted November 2, 2015; accepted for publication February 26, 2016 Dr. Nguyen is a consultant for Ferring. Dr. Feng receives research support from Varian, and is on the advisory board for Medivation
NCCN is accredited as a provider of continuing nursing education by the American Nurses Credentialing Center's Commission on Accreditation.

NCCN designates the education activity for a maximum of 1.0 contact hour. Accreditation as a provider refers to recognition of educational activities only; accredited status does not imply endorsement by NCCN or ANCC of any commercial products discussed/ displayed in conjunction with the educational activity. Kristina M. Gregory, RN, MSN, OCN, is our nurse planner for this educational activity.

All clinicians completing this activity will be issued a certificate of participation. To participate in this journal CE activity: 1) review

and Astellas. The remaining authors have disclosed that they have no financial interests, arrangements, affiliations, or commercial interests with the manufacturers of any products discussed in this article or their competitors.

This work is supported by the Prostate Cancer Foundation, Fitz's Cancer Warriors, David and Cynthia Chapin, Hugh Simons in Honor of Frank and Anne Simons, Scott Forbes and Gina Ventre Fund, Jeffrey Campbell in Honor of Joan Campbell, and a grant from an anonymous family foundation.

Corresondence: Paul L. Nguyen, MD, Brigham and Women's Hospital, 75 Francis Street, Boston, MA 02115. E-mail: pnguyen@LROC.harvard.edu 
Muralidhar et al

the learning objectives and author disclosures; 2) study the education content; 3 ) take the posttest with a $66 \%$ minimum passing score and complete the evaluation at http://education. nccn.org/node/78270; and 4) view/print certificate.

Release date: April 10, 2016; Expiration date: April 10, 2017

\section{Learning Objectives}

Upon completion of this activity, participants will be able to:

- Describe patterns of use of long-term ADT by subcategories of high-risk and unfavorable intermediate-risk prostate cancer

- Explain factors that might be associated with different durations of ADT use in men with high-risk prostate cancer managed with EBRT

\section{Background}

The current NCCN Clinical Practice Guidelines in Oncology (NCCN Guidelines) for Prostate Cancer recommend long-course (2-3 years) androgen deprivation therapy (ADT) for all patients with high- or very high-risk prostate cancer managed with externalbeam radiation therapy (EBRT). ${ }^{1}$ These recommendations are primarily based on data from 2 randomized controlled trials, the first of which was published in 2003, that demonstrated the superiority of 28 to 36 months of ADT over 4 to 6 months of ADT for locally advanced disease. ${ }^{2,3}$ These results have been subsequently extrapolated to all high-risk prostate cancer in the NCCN Guidelines, and this extrapolation was supported in a recently published randomized trial. ${ }^{4}$

Despite these data from randomized trials, many patients do not receive long-term ADT. We recently found that in a tertiary referral care center, genitourinary oncology specialists prescribed less than 2 years of ADT for approximately half of patients with high-risk prostate cancer managed with radiation. ${ }^{5}$ In most cases, physicians stopped ADT early because of patient intolerance of side effects, physician judgment about the aggressiveness of the disease (eg, clinical stage T3a on MRI only as the sole high-risk feature), or patient age/comorbidity, especially given recent controversy around possible cardiovascular toxicity of ADT in patients with preexisting cardiac comorbidity. ${ }^{5-10}$ These results highlight the delicate balance that must be struck between survival, quality of life, and toxicity when prescribing long-course ADT for high-risk prostate cancer. However, this study was limited to only one center and included approximately 300 patients.
In addition, we have recently created and validated a definition for "favorable high-risk" prostate cancer, defined as clinical stage T1c, Gleason score of $4+4=8$, and prostate-specific antigen (PSA) level less than $10 \mathrm{ng} / \mathrm{mL}$, or clinical stage T1c, Gleason score of 6 , and PSA level greater than $20 \mathrm{ng} /$ $\mathrm{mL}$. Among patients managed with radiation, those with favorable high-risk disease have much better outcomes than those with other high-risk disease, and have outcomes similar to those with unfavorable intermediate-risk disease. Despite inclusion with higher-risk patients in guideline recommendations, it is possible that physicians have already been considering some patients with favorable high-risk prostate cancer to have less aggressive disease than those with other high-risk disease, and treating such patients less aggressively, including with shorter-duration ADT when managed with EBRT.

Therefore, we used a national cancer database linked to Medicare insurance claims data to determine patterns of prescribing long-course ADT for high-risk prostate cancer. We also determined the patterns of long-course ADT use among the 3 subcategories of high-risk disease, including favorable high-risk, other high-risk, and very high-risk disease. As a comparison group, we also report the pattern of long-course ADT for the unfavorable subgroup of intermediate-risk disease, for whom short-course ADT is standard. ${ }^{1,11}$ We hypothesized that the 3 subcategories of high-risk disease would have significantly different rates of ADT, with favorable highrisk and unfavorable intermediate-risk disease being treated with similar durations of ADT. In addition, we hypothesized that the rates of long-term ADT use would be low $(<50 \%)$ in all study subgroups.

\section{Methods}

\section{Patient Population}

The SEER database is a population-based cancer registry that collects cancer diagnostic, treatment, and survival data in addition to patient-specific demographic characteristics, covering $28 \%$ of the US population and $97 \%$ of incident cancers. ${ }^{12}$ SEER was linked to Medicare administrative data, which contains insurance claims data for patients older than 65 years enrolled in Medicare. ${ }^{13}$ We extracted clinical and demographic data about patients from SEER, including T stage, Gleason pattern and score, 
PSA level, lymph node status, distant metastasis status, race, marital status, zip code median income level, zip code educational attainment (percentage of adults aged $\geq 25$ years who have completed high school only), geographic region (Midwest, West, Northeast, or South), age at diagnosis, and year of diagnosis. We used Medicare claims data to determine the duration of ADT received by patients (codes C9216, C9430, J0128, J1950, J9202, J1675, J9217-19, J9225-26, J3315, S0133, S0165, and Q2020). Codes that referred to orchiectomy (a currently rare and permanent form of androgen deprivation) or diethylstilbestrol (DES; a rarely used form of hormonal therapy), or codes that were not specific enough to determine a duration of ADT were not included. The total corresponding months of androgen suppression were summed as in previous studies. ${ }^{14-17}$ For example, a patient who received 8 injections of leuprolide acetate, $22.5 \mathrm{mg}$ (3-month dosing), would be considered to have completed 2 years of ADT. We also derived the Charlson comorbidity index from Medicare claims data 1 year before prostate cancer diagnosis and categorized the index as 0 to 1 versus 2 or greater using a previously described and validated algorithm. ${ }^{18}$ This study was approved by the Institutional Review Board at the Dana-Farber Cancer Institute.

We used the SEER-Medicare database to identify 5,524 patients diagnosed in 2004 through 2007 with NOMO unfavorable intermediate-risk or high-risk prostate cancer and managed with EBRT alone. The years of inclusion were selected because Gleason score and PSA level are available in SEER starting in 2004 (allowing risk stratification), and we had access to Medicare claims data until the end of 2009, allowing at least 2 years of follow-up for patients diagnosed in 2007 or earlier. Unfavorable intermediate-risk disease was defined based on the classification recently described by Zumsteg et al, ${ }^{19}$ namely intermediate-risk disease (clinical stage T2b-c, Gleason score of 7, or PSA level 10-20 ng/mL with no high-risk features) with more than one intermediate-risk feature or primary Gleason pattern 4; because of limitations in the availability of data in the SEER database, we were not able to include patients who had $50 \%$ or more positive biopsy cores as the original classification system suggested. Highrisk disease was defined as clinical stage T3 to T4, Gleason score of 8 to 10 , or PSA level of $20 \mathrm{ng} / \mathrm{mL}$ or more. Patients were excluded if they did not have available Gleason score information.

Furthermore, we subclassified high-risk disease according to favorable high-risk (clinical stage T1c, Gleason score of $4+4=8$, PSA level $<10 \mathrm{ng} / \mathrm{mL}$, or clinical stage T1c, Gleason score of 6, PSA level $>20$ $\mathrm{ng} / \mathrm{mL}$ ), other high-risk (clinical stage T3a, Gleason score $8-10$, PSA $>20 \mathrm{ng} / \mathrm{mL}$ without favorable or very high-risk features), and very high-risk (clinical stage T3b-T4 or primary Gleason pattern 5).,20 Because of limitations in the SEER database, we were not able to include patients in the very high-risk classification based on the number of biopsy cores with a Gleason score of 8 to 10 as recommended by NCCN. In addition, to account for possible inaccuracies in the recording of some PSA values in the SEER database, ${ }^{21,22}$ we excluded 223 of 5,747 initial patients $(3.9 \%)$ who had discordant values for PSA and PSA interpretation recorded in SEER (eg, PSA level $<4.0 \mathrm{ng} / \mathrm{mL}$ recorded as "positive/ elevated" or PSA level $>4.0 \mathrm{ng} / \mathrm{mL}$ recorded as "negative/normal"). This approach was based on the observations of Schymura et al. ${ }^{23}$

\section{Statistical Analyses}

Stata/MP 14.0 (StataCorp LP, College Station, TX) was used for all statistical analyses. Baseline characteristics were compared using the KruskalWallis or $\chi^{2}$ test as appropriate. We used competing risks regression to model the time to ADT discontinuation, ${ }^{24}$ with death before completion of long-term ADT treated as a competing risk; hazard ratios greater than 1 represented an increased rate of receiving long-course ADT. To account for possible delays in reporting claims data, patients were not considered to have discontinued ADT at less than 24 months if their last month of ADT coverage was within 2 months of their last follow-up time. Differences in the rate of ADT discontinuation between subgroups of patients were determined after adjusting for year of diagnosis, race, zip code income level (stratified by below or above the median zip code income of $\$ 46,706$ ), zip code percentage of adults older than 25 years having completed high school only (stratified by below or above the median of $26.2 \%$ ), geographic area, patient age, and comorbidity. As a sensitivity analysis, we repeated our comparisons after restricting the population of patients to only those who initiated ADT (ie, received $\geq 1$ dose). 
Muralidhar et al

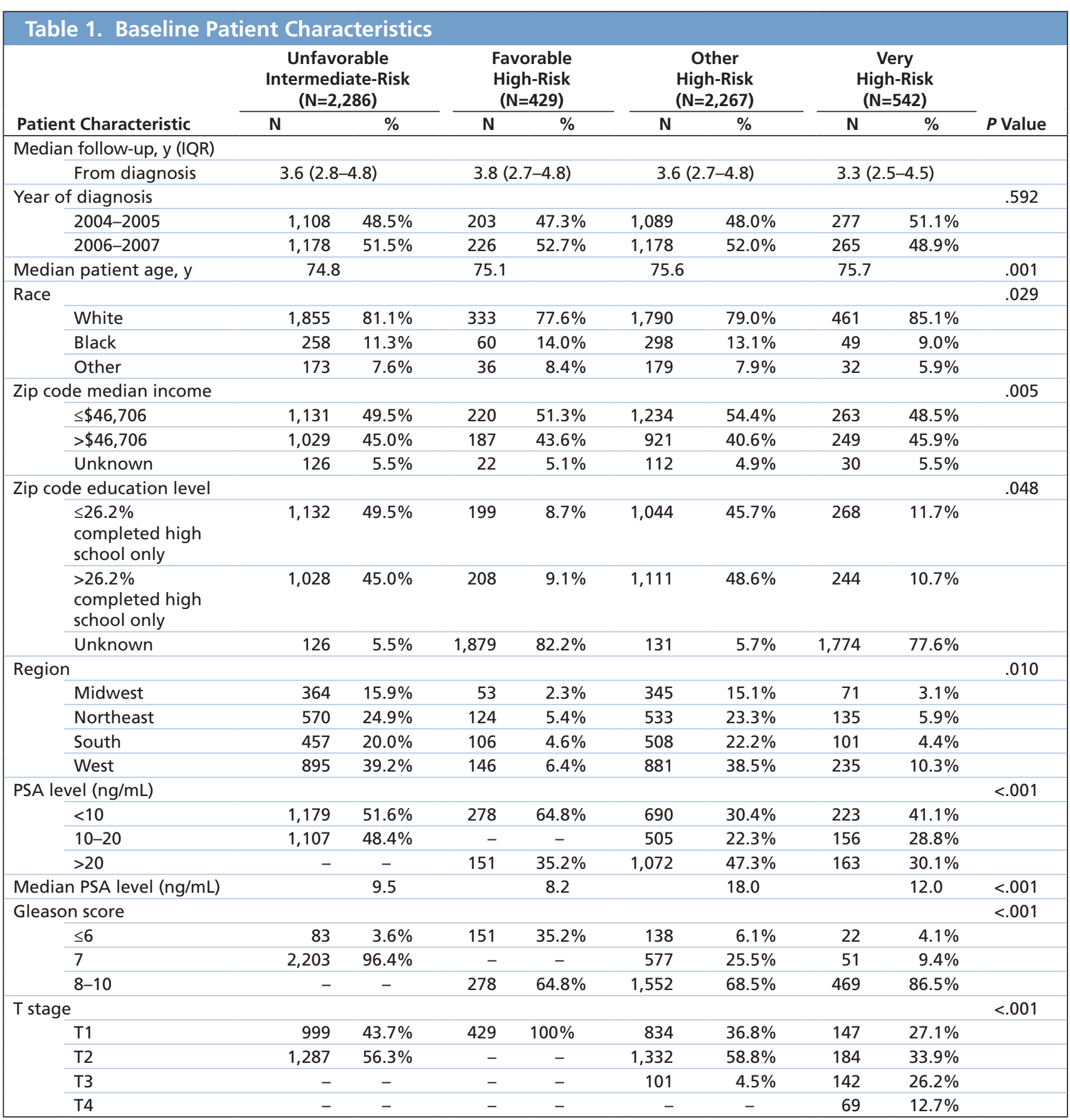

Abbreviations: ADT, androgen deprivation therapy; IQR, interquartile range; PSA, prostate-specific antigen.

\section{Results}

\section{Baseline Patient Characteristics}

Baseline patient characteristics are shown in Table 1. Although statistically significantly different, absolute differences in baseline demographic characteristics among the groups were small. Of patients who received any ADT, 88.9\% received their first dose within 4 months of the recorded date of diagnosis.

\section{Rate of Long-Course ADT Among Patients With Unfavorable Intermediate and High-Risk Prostate Cancer}

Among patients with high-risk disease (ie, clinical stage T3-T4, Gleason score of 8-10, or PSA level 
Nationwide Trends in ADT Use

$>20 \mathrm{ng} / \mathrm{mL}$ ), the rate of long-term ( $\geq 2$ years) ADT was $24.4 \%$. In comparison, the rate of long-term ADT for patients with unfavorable intermediaterisk disease was $12.3 \%$. When adjusting for patient sociodemographic factors and comorbidity level, patients with high-risk disease were significantly more likely to receive long-course ADT than those with unfavorable intermediate-risk disease (adjusted hazard ratio [AHR] 1.62; 95\% CI, 1.53$1.72 ; P<.001)$ (Figure 1). This difference remained significant when only considering the $85.4 \%$ of highrisk patients and $71.4 \%$ of unfavorable intermediaterisk patients who initiated ADT (AHR, 1.43; 95\% CI, 1.34-1.52; $P<.001)$.

In multivariable analysis, increasing patient age was associated with longer-course ADT and later year of diagnosis was associated with receiving shorter durations of ADT (Table 2). Race, marital status, income level, and comorbidity level were not significantly associated with differences in the duration of ADT.

\section{Rate of Long-Course ADT Among}

\section{Subclassifications of High-Risk Prostate Cancer}

To explore the rate of long-term ADT use among subgroups of men with high-risk prostate cancer, we evaluated the 3 subclassifications (favorable, other, and very high) separately (Figure 2). We found that patients with favorable high-risk disease were significantly less likely to receive long-term ADT than those with other high-risk disease $(15.4 \%$ vs $24.6 \%$; AHR, 0.68; 95\% CI, 0.60-0.76; P<.001) and similarly likely as those with unfavorable intermediate-risk disease (15.4\% vs 12.3\%; AHR, 1.10; 95\% CI, 0.98 1.22; $P=.107)$. Those with other high-risk prostate cancer were less likely to receive 2 years of ADT than

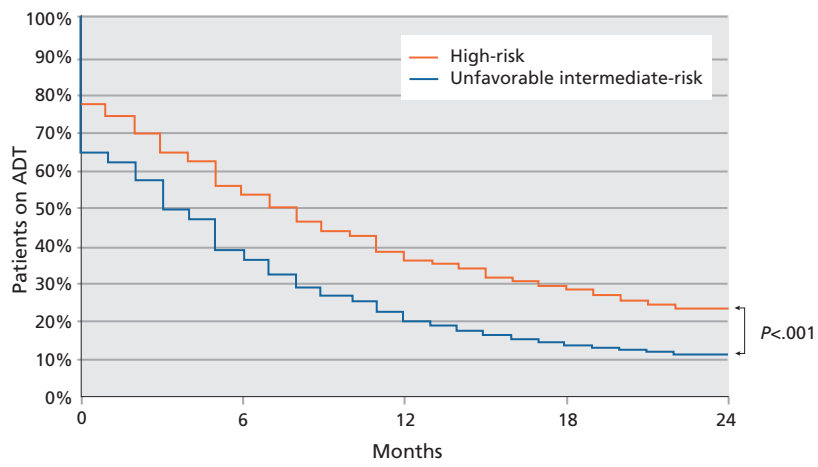

Figure 1. Androgen deprivation therapy (ADT) duration by risk group. High-risk disease includes favorable, other, and very high-risk prostate cancer.

\begin{tabular}{|c|c|c|c|}
\hline & AHR & $95 \% \mathrm{Cl}$ & $P$ Value \\
\hline \multicolumn{4}{|l|}{ Risk group } \\
\hline Intermediate & 1 & & \\
\hline High/very high & 1.60 & $1.51-1.70$ & $<.001$ \\
\hline \multicolumn{4}{|l|}{ Race } \\
\hline White & 1 & & \\
\hline Black & 0.91 & $0.82-1.00$ & .055 \\
\hline Other & 1.00 & $0.90-1.12$ & .975 \\
\hline $\begin{array}{l}\text { Year of diagnosis } \\
\text { (increasing) }\end{array}$ & 0.96 & $0.93-0.98$ & .001 \\
\hline \multicolumn{4}{|l|}{ Marital status } \\
\hline Unmarried & 1 & & \\
\hline Married & 1.02 & $0.95-1.09$ & .643 \\
\hline \multicolumn{4}{|l|}{ Zip code median income } \\
\hline$\leq \$ 46,706$ & 1 & & \\
\hline$>\$ 46,706$ & 0.94 & $0.88-1.01$ & .073 \\
\hline \multicolumn{4}{|l|}{ Zip code education level } \\
\hline $\begin{array}{l}\leq 26.2 \% \text { completed } \\
\text { high school only }\end{array}$ & 1 & & \\
\hline $\begin{array}{l}>26.2 \% \text { completed } \\
\text { high school only }\end{array}$ & 1.03 & $0.96-1.11$ & .415 \\
\hline \multicolumn{4}{|l|}{ Geographic region } \\
\hline Midwest & 1 & & \\
\hline Northeast & 1.03 & $0.93-1.14$ & .546 \\
\hline South & 0.87 & $0.78-0.97$ & .009 \\
\hline West & 0.87 & $0.78-0.96$ & .006 \\
\hline Patient age (increasing) & 1.01 & $1.00-1.01$ & .003 \\
\hline \multicolumn{4}{|l|}{ Comorbidity index } \\
\hline Klabunde/Charlson 0 & 1 & & \\
\hline Klabunde/Charlson 1+ & 1.04 & $0.96-1.01$ & .315 \\
\hline
\end{tabular}

Abbreviations: ADT, androgen deprivation therapy; AHR, adjusted hazard ratio.

those with very high-risk disease $(24.6 \%$ vs $30.8 \%$; AHR, 0.83; 95\% CI, 0.74-0.93; $P=.002$ ).

The rates of initiating ADT among those with favorable high-risk, other high-risk, or very highrisk disease were $75.8 \%, 86.2 \%, 90.2 \%$, respectively. When only patients who initiated ADT were considered, we observed similar relationships (favorable high-risk vs other high-risk: AHR, 0.78; $P=.001$; favorable high-risk vs unfavorable intermediate-risk: AHR, 1.09; $P=.244$; other highrisk vs very high-risk: AHR, 0.84; $P=.009)$. 
Muralidhar et al

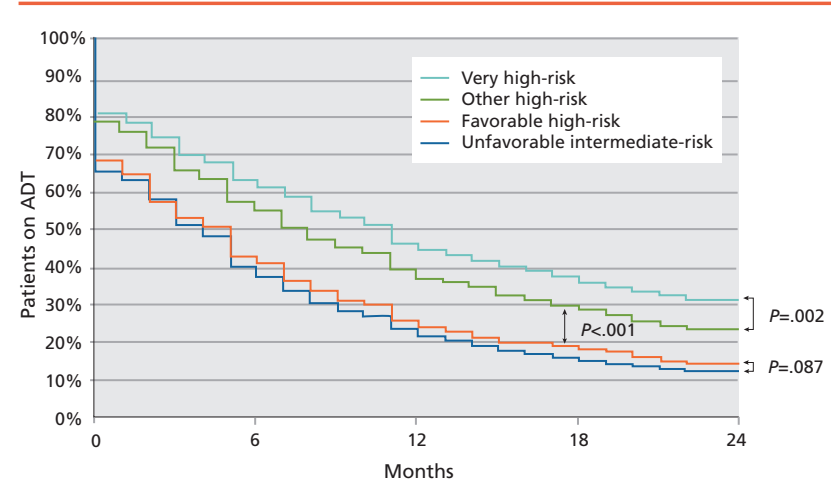

Figure 2. Androgen deprivation therapy (ADT) duration by risk group, including subclassifications of high-risk disease.

\section{Discussion}

In this patterns-of-care study using a large national cancer database linked to Medicare insurance claims, we found that the rate of long-term ( $\geq 2$ years) ADT use among patients with EBRT-managed prostate cancer varied significantly by risk subclassification within high-risk disease, despite NCCN Guidelines recommending similar management $(2-3$ years ADT) for all such patients. In particular, patients with favorable high-risk disease (clinical stage T1c, Gleason score of $4+4=8$, PSA level $<10 \mathrm{ng} / \mathrm{mL}$, or clinical stage T1c, Gleason score of 6, PSA level $>20 \mathrm{ng} / \mathrm{mL}$ ) had much lower rates of long-term ADT use $(15.4 \%)$ than those with other high-risk disease $(24.6 \%)$ and comparable rates as those with unfavorable intermediate-risk disease (12.3\%). On the other hand, patients with very high-risk disease tended to be significantly more likely than other patients to receive long-term ADT (30.8\%). Even when patients who received no ADT at all were excluded, these relationships among these 4 subgroups persisted, suggesting that differences in ADT initiation cannot completely explain these differences in the use of long-term ADT.

Our results suggest that oncologists view the subcategories of high-risk disease to be heterogeneous in disease aggressiveness, thereby warranting different levels of therapy. Although we could not determine the reasons for patients receiving shorter durations of ADT, our findings suggest that physicians might consider favorable high-risk disease to be similarly aggressive as unfavorable intermediate-risk disease, which is typically treated with short-course (4-6 months) ADT when managed with radiation. On the other hand, very highrisk disease is likely viewed as a more aggressive clinical entity that warrants a greater rate of long-term ADT use.
Our findings affirm the current substratification of high risk versus very high-risk prostate cancer in the NCCN Guidelines for Prostate Cancer, ${ }^{1}$ but also suggest that "favorable high risk" be considered a distinct disease subgroup.

The absolute difference in the rates of longterm ADT use between favorable high-risk disease and other high-risk disease was higher than the difference in rates between other high-risk and very high-risk $(9.2 \%$ vs $6.2 \%)$, suggesting the possibility that high-risk disease should be further subclassified into favorable versus other high-risk prostate cancer. Recent data suggest that patients with favorable highrisk disease have much better cancer-specific outcomes than those with other high-risk disease, and similar outcomes as those with unfavorable intermediate-risk prostate cancer. ${ }^{20}$ The data presented here suggest that EBRT-managed favorable high-risk disease and unfavorable intermediate-risk disease are already treated similarly by physicians in the United States.

We also found that despite randomized data published in 2003 from the RTOG 92-02 trial showing an improvement in cancer-specific outcomes with long-term compared with short-term ADT for patients with high-risk prostate cancer, ${ }^{3}$ most high-risk patients in our study did not complete a 2 -year course of ADT. Although one explanation for the relatively low rate of long-term ADT used in our study may be slow adoption of this published data, we did not find any increase in the rate of long-term ADT use associated with increasing year of diagnosis. Alternatively, the somewhat low rates of long-term ADT use may reflect a balancing act between the survival benefit with long-term ADT and the side effects associated with ADT use. In particular, ADT has known side effects related to quality of life, including decreased libido, fatigue, weight gain, and sexual dysfunction. ${ }^{6}$ Given these significant side effects, patient preference may also influence the duration of ADT ultimately received. In addition, controversy exists over whether ADT increases the risk of cardiovascular events, especially in men with preexisting cardiac comorbidity, ${ }^{6,710,25,26}$ although the years included in the present study precede this controversy. Therefore, it may be reasonable for physicians to prescribe shorter courses of ADT for patients who cannot tolerate the side effects or who may be harmed from the various described toxicities. We have recently shown that in a tertiary care referral center, genitourinary 
oncology specialists tended to give less than longterm ADT for approximately half of patients, most often because of intolerance of side effects and patient comorbidity/age. ${ }^{5}$

In multivariable analysis, we found that some patient-specific factors, including race, marital status, income level, and comorbidity level, were not associated with different durations of ADT. The lack of association between these demographic factors and receipt of hormonal therapy is reassuring, particularly because other studies have noted race and socioeconomic treatment disparities in the receipt of radiation or surgery for high-risk prostate cancer. ${ }^{27,28}$ Because everyone in our cohort was treated with EBRT, it is possible that they represent a group of patients that already had access to care, hence eliminating the factors that may otherwise underlie such treatment disparities. This explanation is consistent with prior work suggesting that insurance may mitigate racial disparities in the treatment for high-risk prostate cancer. ${ }^{29}$ Although other work has suggested that increasing comorbidity is associated with an increased probability of stopping ADT before 2 years, ${ }^{5}$ we did not find such an association in the present study. One explanation is that the included years of the present study slightly precede the controversy regarding the possibility of cardiovascular harm from ADT in patients with preexisting cardiac comorbidity, and therefore comorbidity may not have influenced physician decisions for this study cohort.

Our study should be interpreted with respect to its limitations. First, we were limited in the data that we had access to within the SEER database. For example, we were not able to determine the reasons for discontinuation. Although prior work in a single tertiary care referral center has described some of the reasons for receipt of less than 2 years of ADT, future work is needed to study the reasons for receipt of shorter-course ADT in other contexts. In addition, because detailed core biopsy data were not available in SEER for the years of our study, we were not able to include these data in the definitions of unfavorable intermediate-risk ${ }^{19}$ or very high-risk ${ }^{1}$ disease. Therefore, some patients with unfavorable intermediate-risk or very high-risk disease may have been excluded from our study or misclassified as other high-risk, respectively. However, the overall number of patients in this category is likely small and unlikely to affect the overall conclusions of this study.
Second, our risk classification depended on recorded values of PSA in the SEER database, which may contain some errors due to a misplaced decimal point. ${ }^{21,22}$ However, preliminary study by the SEER program has suggested that these errors would cause misclassification of only approximately $5 \%$ of patients within the 3 main categories relevant to this study (PSA $<10 \mathrm{ng} / \mathrm{mL}, 10-20 \mathrm{ng} / \mathrm{mL}$, and $>20 \mathrm{ng} / \mathrm{mL}$ ). Based on the work of others, ${ }^{23}$ we attempted to account for these errors by excluding the $3.9 \%$ of patients in our initial cohort who had PSA values of less than 4 $\mathrm{ng} / \mathrm{mL}$ listed as "positive" and who had PSA values of greater than $4 \mathrm{ng} / \mathrm{mL}$ listed as "negative." Although this approach did not likely account for all possible errors in the SEER database, the errors are not likely to systematically bias our results given the relatively low rate of incorrect classification and random nature of these coding errors.

Third, we only included patients diagnosed from 2004 to 2007 based on the availability of data in the SEER-Medicare database. It is possible that practice patterns changed after the 2009 publication of the EORTC22961 trial, ${ }^{2}$ as was suggested in an institutional series at a single tertiary referral center. ${ }^{5}$ Future work is needed to determine national practice trends after the 2009 publication of this important study.

\section{Conclusions}

Using a large national cancer database linked with Medicare claims information, we found that patients with EBRT-managed high-risk prostate cancer received significantly different durations of ADT based on subclassification of disease. Patients with favorable high-risk prostate cancer received significantly lower rates of long-term ADT than those with other high-risk disease, and similar rates as those with unfavorable intermediate-risk disease. These data suggest that physicians nationwide already consider favorable high-risk and unfavorable intermediate-risk prostate cancer to represent similarly less aggressive forms of the disease compared with other high-risk or very high-risk disease.

\section{References}

1. Mohler JL, Armstrong AJ, Bahnson RR, et al. NCCN Clinical Practice Guidelines in Oncology: Prostate Cancer. Verison 2.2016. Accessed March 9, 2016. To view the most recent version of these guidelines, visit NCCN.org. 
Muralidhar et al

2. Bolla M, de Reijke TM, Van Tienhoven G, et al. Duration of androgen suppression in the treatment of prostate cancer. N Engl J Med 2009;360:2516 2527.

3. Hanks GE, Pajak TF, Porter A, et al. Phase III trial of long-term adjuvant androgen deprivation after neoadjuvant hormonal cytoreduction and radiotherapy in locally advanced carcinoma of the prostate: the Radiation Therapy Oncology Group protocol 92-02. J Clin Oncol 2003;21:3972-3978.

4. Zapatero A, Guerrero A, Maldonado X, et al. High-dose radiotherapy with short-term or long-term androgen deprivation in localised prostate cancer (DART01/05 GICOR): a randomised, controlled, phase 3 trial. Lancet Oncol 2015;16:320-327.

5. Muralidhar V, Regan MM, Werner L, et al. Duration of androgen deprivation therapy for high-risk prostate cancer: application of randomized trial data in a tertiary referral cancer center [published online ahead of print December 17, 2015]. Clin Genitourin Cancer, doi: 10.1016/j.clgc.2015.12.008.

6. Nguyen PL, Alibhai SM, Basaria S, et al. Adverse effects of androgen deprivation therapy and strategies to mitigate them. Eur Urol 2015;67:825-836.

7. Nanda $\mathrm{A}$, Chen $\mathrm{MH}$, Braccioforte $\mathrm{MH}$, et al. Hormonal therapy use for prostate cancer and mortality in men with coronary artery disease-induced congestive heart failure or myocardial infarction. JAMA 2009;302:866-873.

8. Ziehr DR, Chen MH, Zhang D, et al. Association of androgen-deprivation therapy with excess cardiac-specific mortality in men with prostate cancer. BJU Int 2015;116:358-365.

9. Nguyen PL, Je Y, Schutz FA, et al. Association of androgen deprivation therapy with cardiovascular death in patients with prostate cancer: a meta-analysis of randomized trials. JAMA 2011;306:2359-2366.

10. D'Amico AV, Chen MH, Renshaw AA, et al. Androgen suppression and radiation vs radiation alone for prostate cancer: a randomized trial. JAMA 2008;299:289-295.

11. Greene DE, Mayadev JS, Valicenti RK. Radiation treatment for patients with intermediate-risk prostate cancer. Ther Adv Urol 2012;4:113-124.

12. Overview of the SEER Program. National Cancer Institute; Surveillance, Epidemiology, and End Results Program Web site. Available at: http://seer. cancer.gov/about/overview.html. Accessed September 3, 2015.

13. Warren JL, Klabunde CN, Schrag D, et al. Overview of the SEER-Medicare data: content, research applications, and generalizability to the United States elderly population. Med Care 2002;40(8 Suppl):IV-3-18.

14. Shahinian VB, Kuo YF, Freeman JL, Goodwin JS. Risk of fracture after androgen deprivation for prostate cancer. N Engl J Med 2005;352:154-164.

15. Keating NL, O'Malley AJ, Smith MR. Diabetes and cardiovascular disease during androgen deprivation therapy for prostate cancer. J Clin Oncol 2006;24:4448-4456.

16. Schmid M, Hanske J, Ravi P, et al. Relationship between androgen deprivation therapy and community-acquired respiratory infections in patients with prostate cancer [published online ahead of print January 13, 2016]. Int J Urol, doi: 10.1111/iju.13043.

17. Schmid M, Sammon JD, Reznor G, et al. Dose-dependent effect of androgen deprivation therapy for localized prostate cancer on adverse cardiac events [published online ahead of print June 13, 2015]. BJU Int, doi: 10.1111/bju.

18. Klabunde CN, Potosky AL, Legler JM, Warren JL. Development of a comorbidity index using physician claims data. J Clin Epidemiol 2000;53:12581267.

19. Zumsteg ZS, Spratt DE, Pei I, et al. A new risk classification system for therapeutic decision making with intermediate-risk prostate cancer patients undergoing dose-escalated external-beam radiation therapy. Eur Oncol 2013;64:895-902.

20. Muralidhar V, Chen MH, Reznor G, et al. Definition and validation of "favorable high-risk prostate cancer": implications for personalizing treatment of radiation-managed patients. Int J Radiat Oncol Biol Phys 2015;93:828-835.

21. PSA Values and SEER Data. National Cancer Institute: Surveillance, Epidemiology, and End Results Program. Available at: http://seer.cancer.gov/ data/psa-values.html. Accessed September 3, 2015.

22. Furlow B. US National Cancer Institute investigates PSA coding errors. Lancet Oncol 2015;16:614.

23. Schymura MJ, Sun L, Percy-Laurry A. Prostate cancer collaborative stage data items--their definitions, quality, usage, and clinical implications: a review of SEER data for 2004-2010. Cancer 2014;120(Suppl 23):3758-3770.

24. Fine JP, Gray RJ. A proportional hazards model for the subdistribution of a competing risk. J Am Stat Assoc 1999;94:496-509.

25. Efstathiou JA, Bae K, Shipley WU, et al. Cardiovascular mortality and duration of androgen deprivation for locally advanced prostate cancer: analysis of RTOG 92-02. Eur Urol 2008;54:816-823.

26. Alibhai SM, Duong-Hua M, Sutradhar R, et al. Impact of androgen deprivation therapy on cardiovascular disease and diabetes. J Clin Oncol 2009;27:34523458.

27. Ziehr DR, Mahal BA, Aizer AA, et al. Income inequality and treatment of African American men with high-risk prostate cancer. Urol Oncol 2015;33:18 e7-3.

28. Mahal BA, Aizer AA, Ziehr DR, et al. Trends in disparate treatment of African American men with localized prostate cancer across National Comprehensive Cancer Network risk groups. Urology 2014;84:386-392.

29. Mahal BA, Ziehr DR, Aizer AA, et al. Getting back to equal: the influence of insurance status on racial disparities in the treatment of African American men with high-risk prostate cancer. Urol Oncol 2014;32:1285-1291.

\section{Instructions for Completion}

To participate in this journal CE activity: 1) review the learning objectives and author disclosures; 2 ) study the education content; 3 ) take the posttest with a $66 \%$ minimum passing score and complete the evaluation at http://education.nccn.org/ node/78270; and 4) view/print certificate. After reading the article, you should be able to answer the following multiple- choice questions. Credit cannot be obtained for tests completed on paper. You must be a registered user on NCCN.org. If you are not registered on NCCN.org, click on "New Member? Sign up here" link on the left hand side of the Web site to register. Only one answer is correct for each question. Once you successfully answer all posttest questions you will be able to view and/or print your certificate. Software requirements: Internet

\section{Posttest Questions}

1. True or False: Long-term ( $\geq 2$ years) ADT use among patients with high-risk EBRT-managed prostate cancer varied significantly by risk subclassification, despite NCCN Guidelines recommending similar management ( $2-3$ years ADT) for all such patients.

2. has been defined as clinical stage T1C, Gleason score of $4+4=8$, and PSA $<10 \mathrm{ng} / \mathrm{mL}$, or clinical stage T1c, Gleason score of 6 , and PSA $>20 \mathrm{ng} / \mathrm{mL}$.

a. Very high-risk b. Favorable high-risk

c. Unfavorable intermediate-risk

d. Other high-risk

3. Which of the following factors might be associated with different durations of ADT?

a. Patient intolerance to side effects

b. Physician judgement regarding aggressiveness of disease

c. Patient age or comorbidity

d. All of the above 